\title{
Features of hand-foot crawling behavior in human
}

adults

M. J. MacLellan, Y. P. Ivanenko, G. Cappellini, F. Sylos Labini and F. Lacquaniti

J Neurophysiol 107:114-125, 2012. First published 5 October 2011; doi:10.1152/jn.00693.2011

You might find this additional info useful...

This article cites 58 articles, 20 of which can be accessed free at:

/content/107/1/114.full.html\#ref-list-1

This article has been cited by 2 other HighWire hosted articles

Function dictates the phase dependence of vision during human locomotion

David Logan, Yuri P. Ivanenko, Tim Kiemel, Germana Cappellini, Francesca Sylos-Labini,

Francesco Lacquaniti and John J. Jeka

J Neurophysiol, July 1, 2014; 112 (1): 165-180.

[Abstract] [Full Text] [PDF]

Changes in the Spinal Segmental Motor Output for Stepping during Development from Infant to Adult

Yuri P. Ivanenko, Nadia Dominici, Germana Cappellini, Ambrogio Di Paolo, Carlo Giannini, Richard E. Poppele and Francesco Lacquaniti

J. Neurosci., February 13, 2013; 33 (7): 3025-3036.

[Abstract] [Full Text] [PDF]

Updated information and services including high resolution figures, can be found at:

/content/107/1/114.full.html

Additional material and information about Journal of Neurophysiology can be found at: http://www.the-aps.org/publications/jn

This information is current as of December 4, 2014.

Journal of Neurophysiology publishes original articles on the function of the nervous system. It is published 12 times a year (monthly) by the American Physiological Society, 9650 Rockville Pike, Bethesda MD 20814-3991. Copyright @ 2012 by the American Physiological Society. ISSN: 0022-3077, ESSN: 1522-1598. Visit our website at http://www.the-aps.org/. 


\title{
Features of hand-foot crawling behavior in human adults
}

\author{
M. J. MacLellan, ${ }^{1}$ Y. P. Ivanenko, ${ }^{1}$ G. Cappellini, ${ }^{1}$ F. Sylos Labini, ${ }^{1}$ and F. Lacquaniti ${ }^{1,2,3}$ \\ ${ }^{1}$ Laboratory of Neuromotor Physiology, Santa Lucia Foundation, Rome; ${ }^{2}$ Center of Space Bio-medicine and ${ }^{3}$ Department of \\ Neuroscience, University of Rome Tor Vergata, Rome, Italy
}

Submitted 25 July 2011; accepted in final form 30 September 2011

MacLellan MJ, Ivanenko YP, Cappellini G, Sylos Labini F, Lacquaniti F. Features of hand-foot crawling behavior in human adults. J Neurophysiol 107: 114-125, 2012. First published October 5, 2011; doi:10.1152/jn.00693.2011.-Interlimb coordination of crawling kinematics in humans shares features with other primates and nonprimate quadrupeds, and it has been suggested that this is due to a similar organization of the locomotor pattern generators (CPGs). To extend the previous findings and to further explore the neural control of bipedal vs. quadrupedal locomotion, we used a crawling paradigm in which healthy adults crawled on their hands and feet at different speeds and at different surface inclinations $\left(13^{\circ}, 27^{\circ}\right.$, and $35^{\circ}$ ). Ground reaction forces, limb kinematics, and electromyographic (EMG) activity from 26 upper and lower limb muscles on the right side of the body were collected. The EMG activity was mapped onto the spinal cord in approximate rostrocaudal locations of the motoneuron pools to characterize the general features of cervical and lumbosacral spinal cord activation. The spatiotemporal pattern of spinal cord activity significantly differed between quadrupedal and bipedal gaits. In addition, participants exhibited a large range of kinematic coordination styles (diagonal vs. lateral patterns), which is in contrast to the stereotypical kinematics of upright bipedal walking, suggesting flexible coupling of cervical and lumbosacral pattern generators. Results showed strikingly dissimilar directional horizontal forces for the arms and legs, considerably retracted average leg orientation, and substantially smaller sacral vs. lumbar motoneuron activity compared with quadrupedal gait in animals. A gradual transition to a more vertical body orientation (increasing the inclination of the treadmill) led to the appearance of more prominent sacral activity (related to activation of ankle plantar flexors), typical of bipedal walking. The findings highlight the reorganization and adaptation of CPG networks involved in the control of quadrupedal human locomotion and a high specialization of the musculoskeletal apparatus to specific gaits.

quadrupedal locomotion; arm-leg coordination; electromyographic activity; spinal cord

ALTHOUGH BIPEDAL LOCOMOTION is a hallmark of humans, they can also walk on all four limbs. Toddlers often crawl before they begin walking unsupported; most of them crawl on the hands and knees, but some use a hands-and-feet pattern (Hildebrand 1967). Moreover, a few families have been identified with congenital central nervous system (CNS) disorders resulting in equilibrium impairment and a preferred locomotion on hands and feet (Tan 2010).

Crawling is a form of locomotion that can be used by adults, but few studies have examined this behavior. Most of these studies have analyzed the kinematic, temporal, and stability features of crawling on hands and knees (Babic et al. 2001; Wannier et al. 2001; Webb and Sparrow 2007) and hands and feet (Getchell et al. 2001; Hildeband 1967; Sparrow 1989;

Address for reprint requests and other correspondence: M. J. MacLellan, Laboratory of Neuromotor Physiology, IRCCS Fondazione Santa Lucia, 306 via Ardeatina, 00179 Rome, Italy (e-mail: m.maclellan@hsantalucia.it).
Sparrow and Newell 1994; Webb and Sparrow 2007). Even fewer studies have examined muscle activity during handsand-knees crawling in a limited number of muscles (de Seze et al. 2008; Gallagher et al. 2011). Patrick et al. (2009) performed a thorough coordination analysis of crawling on hands and knees, as well as on hands and feet, in human adults and children. They found a large variability of limb coordination, suggesting flexible underlying control mechanisms. They concluded that humans may have an underlying neural circuitry similar to that of other primates and nonprimate quadrupeds, since many features of interlimb coordination are shared between these groups.

The coordination between the upper and lower limbs in humans may be due to sustained neural connections between the cervical and lumbosacral pattern generators (Wannier et al. 2001; Zehr et al. 2009). Such connections between the upper and lower limb neural controllers have been shown in humans by studying the modulation of electromyographic (EMG) and reflex activity of upper limbs muscles during rhythmic activity of lower limbs muscles and vice versa (Zehr et al. 2009), as well as by showing a tight coordination between the limbs in a variety of locomotor tasks (Dietz and Michel 2009; Donker et al. 2002; Ivanenko et al. 2005). Coupling between the upper and lower limbs has also received significant attention in the context of locomotor impairments (Crenna et al. 2008; Dietz 2002; Ferris et al. 2006; Klimstra et al. 2009; Tan 2010).

Taking the above considerations into account, we wondered whether reconstructing the spatiotemporal organization of the spinal motor output (by mapping muscle activations to the motoneuron pools) would provide insight into the coordination between the cervical (arm muscle control) and lumbosacral (leg muscle control) enlargements during quadrupedal and bipedal gaits in humans. We detailed the spatiotemporal characteristics of kinematic and muscle activation patterns when healthy adults crawled on their hands and feet at different speeds and at different support surface inclinations to generate a repertoire for comparison with human bipedal walking and quadrupedal walking in animals. The results show some unique features of quadrupedal gait performance in humans, including a flexible coupling between cervical and lumbosacral enlargement activity, differences between ground reaction force profiles between the upper and lower limbs, and the level of motoneuron activation in the spinal cord.

\section{METHODS}

Two different groups of participants volunteered for two separate experimental sessions. Ten healthy adults $[7$ males and 3 females, $32.6 \pm 8.9 \mathrm{yr}$ of age $($ mean $\pm \mathrm{SD}), 74.6 \pm 13.8 \mathrm{~kg}$ ] volunteered for the first session, and nine healthy adults ( 5 males and 4 females, $32.9 \pm$ $8.3 \mathrm{yr}$ of age, $67.6 \pm 9.6 \mathrm{~kg}$ ) volunteered for the second session, with 
six participants common to both sessions. The studies were in accordance with the Declaration of Helsinki, and informed consent was obtained from all participants according to our procedures, which were approved by the Ethics Committee at the Santa Lucia Foundation.

\section{Experimental Setup}

The subjects participated in two experimental sessions: 1) treadmill locomotion at different speeds and inclinations and 2) overground locomotion at normal, slow, and fast speeds.

Session 1. Two experimental conditions were performed in this session. The first condition was level crawling or normal erect walking on a treadmill (EN-TRED; Enraf Nonius, Rotterdam, The Netherlands) at speeds ranging from 0.5 to $3.0 \mathrm{~km} / \mathrm{h}$ in increments of $0.5 \mathrm{~km} / \mathrm{h}$ (normal walking was also collected at 2,3 , and $5 \mathrm{~km} / \mathrm{h}$ ) with a rest period of $\sim 3$ min between trials. Participants were given time to adapt to each condition and provided a verbal signal to start collection. Each collection consisted of 10 consecutive strides. We also recorded crawling when the speed of the treadmill was ramped from 0.5 to $3 \mathrm{~km} / \mathrm{h}$ in $25 \mathrm{~s}$ (ramp speed condition). The maximal speed $(3 \mathrm{~km} / \mathrm{h})$ was chosen to correspond approximately to a comfortable and natural speed for crawling. The second condition consisted of crawling on the treadmill at $2 \mathrm{~km} / \mathrm{h}$ with modest inclinations of $13^{\circ}$, $27^{\circ}$, and $35^{\circ}$ (which avoided slippage of the feet on the treadmill surface). Again, participants provided a signal to start the trial, and 10 consecutive strides were collected. All participants performed the level crawling and the $13^{\circ}$ inclination conditions, whereas 6 of 10 participants also performed the $27^{\circ}$ and $35^{\circ}$ inclinations. The total duration of the session was $\sim 1.5 \mathrm{~h}$. All participants were instructed to crawl on their hands and feet (as opposed to crawling on the hands and knees; see Gallagher et al. 2011; Patrick et al. 2009; Yokochi et al. 1990).

Session 2. Participants crawled overground at self-paced normal, slow, and fast speeds while ground reaction forces and kinematics data were recorded. The crawling direction was oriented such that only ipsilateral (right side) ground reaction forces were recorded (Fig. 1B). For these trials, participants crawled at self-selected normal, slow, and fast speeds for 3 trials each or until there complete ground contacts were collected with the right forelimbs and hindlimbs.

\section{Data Recording}

Kinematics data were recorded with a 9-camera Vicon-612 system (Oxford, UK) at $100 \mathrm{~Hz}$. Infrared reflective markers (diameter $1.4 \mathrm{~cm}$ ) were placed bilaterally on the following anatomic landmarks: first distal phalanx, fifth metatarsal head, heel, lateral malleolus, lateral femoral epicondyle, greater trochanter, acromioclavicular joint, lateral humeral epicondyle, ulnar process, and distal phalanx of middle finger (Fig. 1A). All marker data were low-pass filtered offline using a second-order dual-pass Butterworth filter with a cutoff of $7 \mathrm{~Hz}$.

Electromyographic (EMG) activity was recorded at 2,000 Hz using a Delsys Trigno EMG system from the following 26 muscles on the right side of the body: long head of biceps brachii, long head of triceps brachii, anterior deltoid, posterior deltoid, pectoralis major, latissimus dorsi, brachioradialis, extensor carpi ulnaris, extensor carpi radialis, flexor carpi ulnaris, flexor carpi radialis, flexor digitorium superficialis, gluteus maximus, gluteus medius, tensor fascia latte, sartorius, adductor longus, rectus femoris, vastus lateralis, vastus medialis, semitendinosis, biceps femoris, lateral gastrocnemius, medial gastrocnemius, soleus, and tibialis anterior. Electrode placements were performed according to recommendations about how to selectively activate each muscle (Kendall et al. 1993) to optimize the EMG signal and minimize cross talk from adjacent muscles. All EMG data were initially high-pass (30-Hz cutoff) filtered offline using a second-order dual-pass Butterworth filter and bandstop filtered at a frequency of 50 Hz. EMG data were then full-wave rectified and low-pass filtered using a second-order dual-pass (zero phase) Butterworth filter with a cutoff of $10 \mathrm{~Hz}$.

Ground reaction forces were collected (session 2) with a force plate $(0.9 \times 0.6-\mathrm{m}$ 9287B; Kistler, Zurich, Switzerland $)$ at 1,000 Hz. All ground reaction force data were low-pass filtered offline using a second-order dual-pass Butterworth filter with a cutoff of $20 \mathrm{~Hz}$, normalized to participant body weight, and time-interpolated to 100 points for interstride averaging.

\section{Data Analysis}

The recorded kinematics data were used to construct a linked segment model consisting of the trunk, upper arm, forearm, hand, thigh, shank, and foot bilaterally. This model was used to calculate joint angles on the right side of the body at the wrist, elbow, and shoulder in the upper limb and the ankle, knee, and hip in the lower limb (Fig. 1A, arrows indicate positive joint motion). Cycle durations of the upper and lower limbs were determined by two consecutive right wrist contacts and first distal phalanx contacts, respectively (the first parts of the body to make contact with the ground during crawling). Swing duration of the upper and lower limbs was determined from lift off of the right distal phalanx of middle finger to wrist contact and lift off of the right first distal phalanx to first distal phalanx contact, respectively. Excursions of the upper and lower limbs were determined by the distance traveled by the right wrist and first distal phalanx, respectively, during swing. Ipsilateral phase lag (IPL) between upper and lower limbs was determined using the methods described by Patrick et al. (2009). In brief, the relative timing of right upper limb contact was expressed as a percentage of the gait cycle determined by consecutive right foot contacts:

$$
\mathrm{IPL}=\frac{t_{1}}{T} \cdot 100 \%
$$

where $t_{1}$ is interval of time between right foot and right hand touchdown events and $T$ is cycle duration (Fig. 2A). According to this method, lateral gait patterns (ipsilateral upper/lower limb contact at similar instances) are determined at a value of $0 \%$ and diagonal gait patterns (contralateral upper/lower limb contact at similar instances) are determined at a value of $50 \%$. Intermediate values $(\sim 25 \%)$ correspond to no limb pairing.

The orientation of the upper limb (shoulder to point of contact, acromioclavicular joint-distal phalanx of middle finger) relative to a

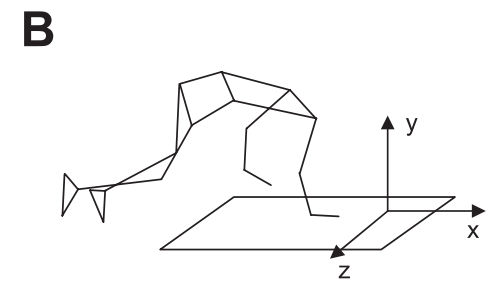

Fig. 1. Experimental setup. A: a 3-dimensional stick diagram of treadmill crawling with marker positions and identification of joint angle measurements with arrows indicating positive joint motion. $B$ : illustration of overground crawling orientation with respect to the force plate for collection of ground reaction forces. The crawling direction was oriented such that only ipsilateral (right side) ground reaction forces were recorded. 
A

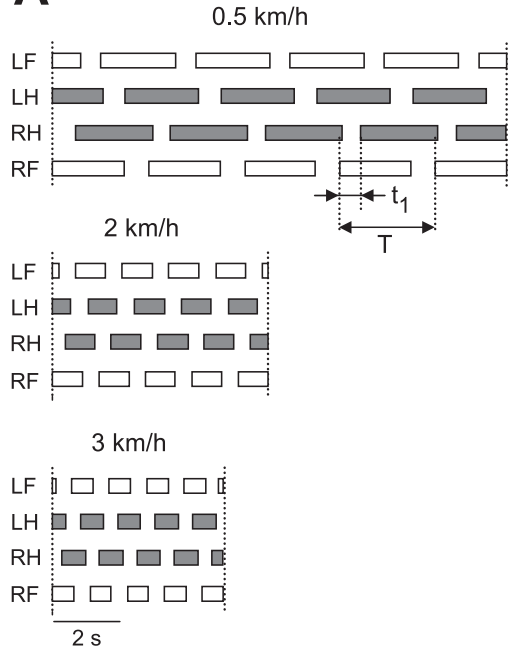

B

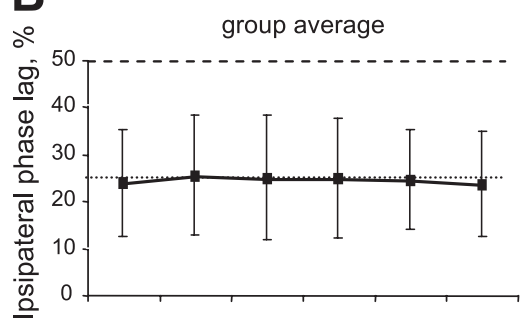

จำ

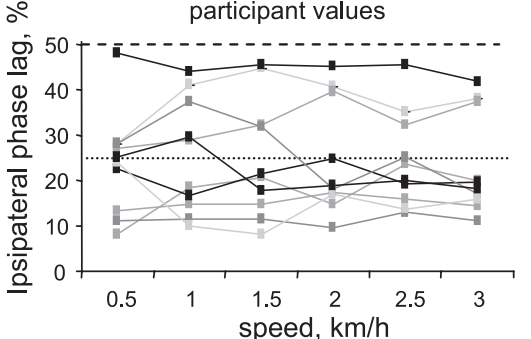

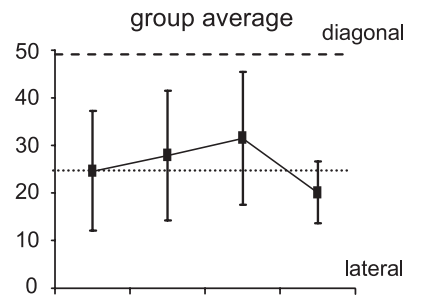

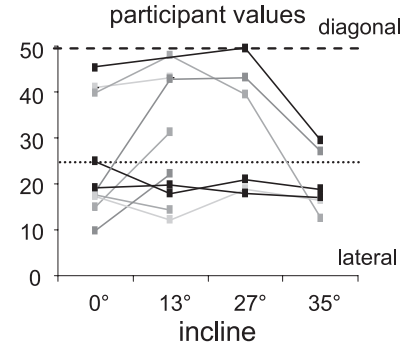

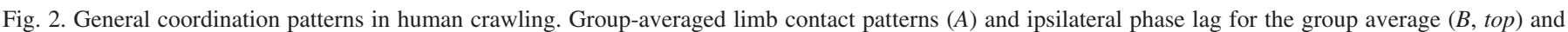

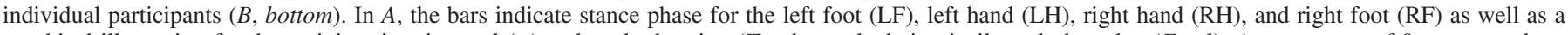

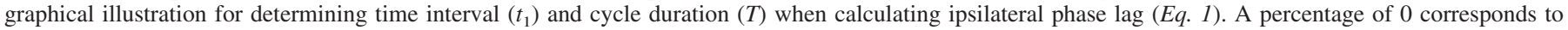
a lateral form of locomotion, whereas a value of $50 \%$ corresponds to diagonal.

vertical line through the proximal joint center at both touchdown and liftoff of a step were computed. Similarly, this was done for the lower limb (hip to contact point: greater trochanter-fifth metatarsal head). Vertical displacement of the hip (greater trochanter) and shoulder (acromioclavicular) joints were calculated as the vertical range over a stride cycle. Average limb orientation was determined for each step by calculating the difference between the limb angle at touchdown and at liftoff and then dividing this number by two. This midpoint of the total limb excursion defines the axis about which the limb oscillates during support phase, as well as allowing comparison with the same angle in selected animals (Larson and Stern 2009). An average limb angle greater than zero indicates a protracted limb axis, whereas values less than zero indicate a retracted limb axis.

Ground reaction force profiles were obtained from the right hand and foot and normalized to participant body weight. The axes of the force plate were aligned such that positive ground reaction forces were directed in the upward (vertical) and forward (horizontal) directions. For graphical purposes, ground reaction force data were time normalized and averaged across participants. Peak force was determined from the maximum (and minimum for horizontal forces) values, and the impulse was calculated by integrating the force-time curve.

\section{Spatiotemporal Patterns of Motoneuron Activity in the Spinal Cord}

The recorded patterns of EMG activity were mapped onto the approximate rostrocaudal location of motoneuron (MN) pools in the human spinal cord (Ivanenko et al. 2006). This approach provides an interpretation of the output from locomotor central pattern generators (CPGs) in terms of segmental control rather than in terms of individual muscle control (Yakovenko et al. 2002). This approach can be used to characterize network architecture for different gaits by considering relative intensities, spatial extent, and temporal structure of the spinal motor output (Ivanenko et al. 2008).

In this study, we used two myotomal charts: those of Kendall et al. (1993) and those of Sharrard (1964). Kendall et al. compiled reference segmental charts for all body muscles by combining the anatomic and clinical data from six different sources. A capital X in Kendall's chart denotes localization agreed on by five or more sources, a lowercase $\mathrm{x}$ denotes agreement of three to four sources, and a bracketed (x) denotes agreement of only two sources. In our maps, $\mathrm{X}$ and $\mathrm{x}$ were weighted 1 and 0.5 , respectively, whereas we discarded (x). We assumed that our population of subjects had the same spinal topography as this reference population.

To reconstruct the output pattern of any given spinal segment $S_{j}$ of the most active lumbosacral segments (L2-S2), we averaged all rectified EMG waveforms corresponding to that segment (Cappellini et al. 2010; Ivanenko et al. 2008):

$$
S_{j}=\frac{\sum_{i=1}^{n_{j}} k_{i j} \cdot \mathrm{EMG}_{i}}{n_{j}}
$$

where $n_{j}$ is the number of $\mathrm{EMG}_{i}$ waveforms corresponding to the $j$ th segment, $k_{i j}$ is the weighting coefficient for the $i$ th muscle (X and $\mathrm{x}$ in Kendall's chart were weighted with $k_{i j}=1$ and $k_{i j}=0.5$, respectively). The assumption implicit in this method is that the rectified EMG provides an indirect measure of the net firing of MNs of that muscle. EMGs were expressed in microvolts and normalized to the physiological cross-sectional area (PCSA) of lower limb (Ward et al. 2009) and upper limb muscles (Ramsay et al. 2009; Wood et al. 1989). To this end, the contribution of each muscle to the estimated activity of the $j$ th segment (Eq. 2) was multiplied by its PCSA (Cappellini et al. 2011).

We used the Kendall chart results in the six lumbosacral (L2-S2) and five cervical (C5-T1) discrete activation waveforms to examine the output from the main enlargements of the spinal cord. The Kendall chart indicates only the segments innervating each muscle and not the fraction of total motor pool of the muscle that can be assigned to a segment. For comparison with previously published cat data that include a greater spatial resolution (Yakovenko et al. 2002), we also used the Sharrard (1964) data table for innervation, which approximates the proportion of total muscle activation attributable to each segment (by taking multiple slices within each spinal segment), instead of assuming equal proportions in all segments. To this end, we subdivided each segment into six subsegments and applied the same equation (Eq. 2) for each jth subsegment (Ivanenko et al. 2006). The resulting spinal cord maps of activation were not smoothed but contained 36 discrete bands (6 subsegments $\times 6$ segments; L2-S2). The data analysis and spinal MN activity map construction were performed with software written in Matlab (R2008; The MathWorks). 
To compare neural activation with crawling kinematics, we performed a cross-correlation between the dominant $\mathrm{MN}$ activity in the lumbar (sum of activity from L2 and L3) and cervical enlargements (sum of activity from $\mathrm{C} 8$ and $\mathrm{T} 1$ ). To determine the phase shift between lumbar and cervical activity, we constructed an algorithm to find the largest correlation coefficient (which was generally above 0.8 ) and expressed the phase shift as a percentage of the gait cycle.

\section{Statistics}

Descriptive statistics include means \pm SD. Repeated-measures ANOVA was used to assess the effect of speed and crawling conditions on gait and motor pattern parameters as well as differences between upper and lower limbs. Paired $t$-tests were used to determine differences between upper and lower limbs for peak forces and impulses, and a one-tailed $t$-test was used to determine whether average limb angle differed from zero. A Pearson correlation was used to test statistically the relationship between lumbar-cervical activity phase shift and IPL. Reported results are considered significant for $P<0.05$.

\section{RESULTS}

\section{Basic Gait Parameters}

Figure $2 A$ illustrates a typical footfall pattern in human crawling. In general, four, three, or two limbs were simultaneously supporting body weight depending on the speed and the instant of the gait cycle. Subjects tended to use a lateral footfall sequence when crawling over the studied range of speeds, i.e., the footfall of a lower limb usually followed that of the ipsilateral upper limb. We quantified this coordination pattern (Fig. 2B) by computing the contact of the right upper limb expressed as a percentage of the gait cycle (Patrick et al. 2009). The results of the IPL analysis between the upper and lower limbs did not differ significantly as a function of crawling speed $\left[F_{(5,45)}=0.204, P=0.87\right.$, Fig. $2 B$, left $]$ or inclination $\left[F_{(3,12)}=1.406, P=0.30\right.$, Fig. $2 B$, right $]$, although a range of different IPL values was observed across participants. Overall, participants showed no limb pairing (IPL 25\%). However, there were participants who exhibited a more "diagonal" (IPL close to $50 \%$ ) or "lateral" pattern (IPL close to $0 \%$ ), and this pattern did not change between crawling conditions (Fig. 2).

As expected, cycle duration decreased $\left[F_{(5,45)}=113.558\right.$, $P<0.001]$ and percentage of swing time increased significantly $\left[F_{(5,45)}=109.210, P<0.001\right]$ with changes in treadmill speed (Fig. 3A). Although cycle duration did not differ significantly between the upper and lower limbs $\left[F_{(1,9)}=0.874, P=\right.$ 0.37], swing duration was consistently shorter in the upper compared with the lower limbs $\left[F_{(1,9)}=13.96, P=0.005\right]$. Moreover, limb excursion increased significantly with treadmill speed $\left[F_{(5,45)}=10.875, P=0.002\right]$ and was systematically larger in the upper limb $\left[F_{(1,9)}=8.897, P=0.015\right]$, consistent with longer stance durations for the arms.

Changing the inclination of the crawling surface had no significant effect on cycle duration $\left[F_{(3,15)}=0.174, P=0.77\right]$, percentage of swing duration $\left[F_{(3,15)}=0.359, P=0.58\right]$, or limb excursion $\left[F_{(3,15)}=0.236, \stackrel{P}{P}=0.70\right]$, and differences were not evident between upper and lower limbs for cycle $\left[F_{(1,5)}=1.007, P=0.36\right]$, swing duration $\left[F_{(1,5)}=0.317, P=\right.$ 0.64], or limb excursion $\left[F_{(1,5)}=0.084, P=0.78\right]$ (Fig. 3B).

Figure 4 illustrates kinematic patterns in human crawling on hands and feet. Vertical greater trochanter (hip) displacements

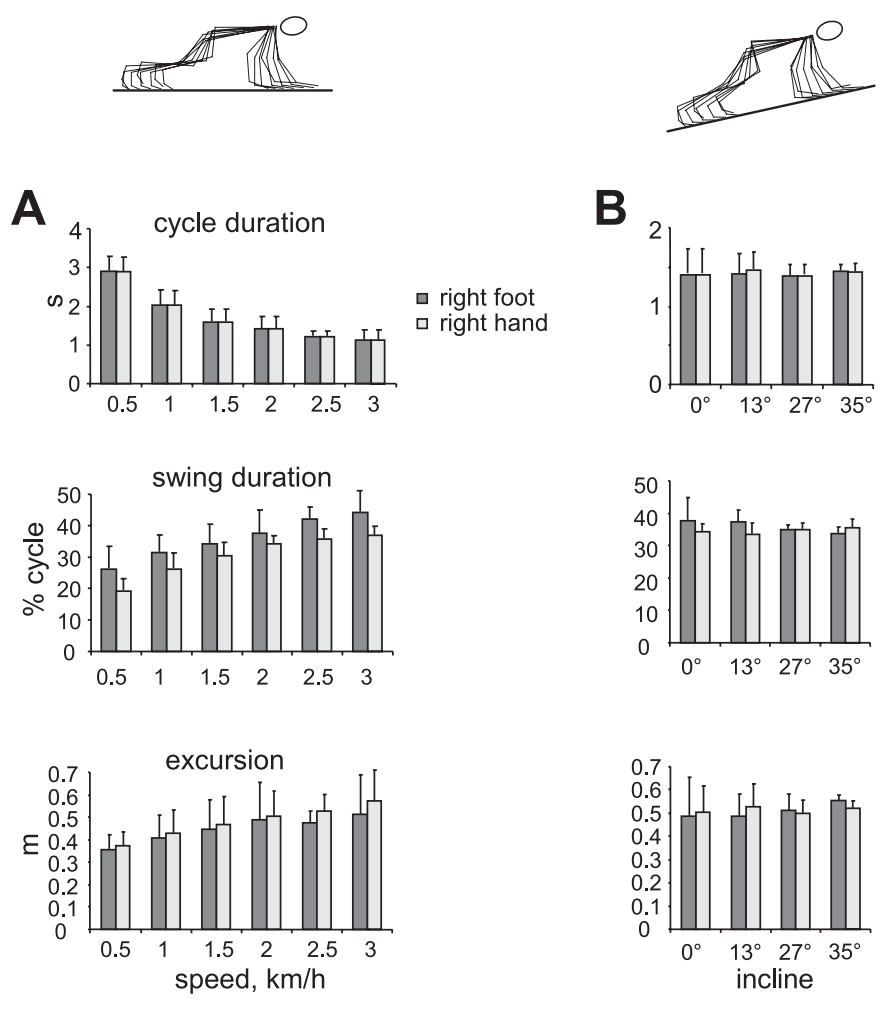

Fig. 3. Mean general gait parameters $( \pm S D)$ during crawling at different treadmill speeds $(A)$ and surface inclinations $(B)$. Stick figures illustrate body positions for 1 gait cycle.

were significantly higher $\left[F_{(1,7)}=34.192, P<0.001\right]$ than those of the acromioclavicular joint (shoulder) at all speeds, although no significant differences were observed between treadmill speeds $\left[F_{(5,35)}=0.554, P=0.73\right]$ (Fig. $4, A$ and $B$, top). Among the joint angles, the shoulder $\left[F_{(5,35)}=40.035\right.$, $P<0.001]$ and elbow $\left[F_{(5,35)}=4.939, P=0.021\right]$ joint angle ranges increased with speed, whereas speed effects were not evident at all other joints $(P>0.05)$ (Fig. 4, $A$ and $B$, bottom).

The orientation of the lower limb differed greatly from that of the upper limb (Table 1). The lower limb was greatly retracted for all crawling speeds $\left[F_{(1,8)}=70.221, P<0.001\right]$. In contrast, the upper limb became more retracted as crawling speed increased $\left[F_{(5,40)}=29.590, P<0.001\right]$ with a $15 \pm 2^{\circ}$ protraction at $0.5 \mathrm{~km} / \mathrm{h}$ and a $9 \pm 2^{\circ}$ protraction at $3 \mathrm{~km} / \mathrm{h}$, whereas no significant changes were found for the retraction of the lower limb $\left[F_{(5,45)}=0.474, P=0.64\right]$.

Human crawling on feet is characterized by distinct patterns of propulsive forces for the lower limbs and braking forces for the upper limbs (Fig. 5A). Comparisons of peak force indicated greater vertical force in the lower compared with the upper limb $(P<0.001)$ and significantly greater peak positive and negative forces for the lower and upper limbs, respectively $(P<0.001$ in both cases) (Fig. 5B). The propulsive and braking function of the legs and arms, respectively, became even more obvious if positive and negative impulses of shear forces were compared: the positive thrust for the legs was on average 10 times greater than the negative one; for the arms, the difference was even larger (15 times). Over a wide range of crawling speeds (Fig. 5C), one can see that peak vertical and horizontal forces tend to increase with crawling speed in the lower 
A
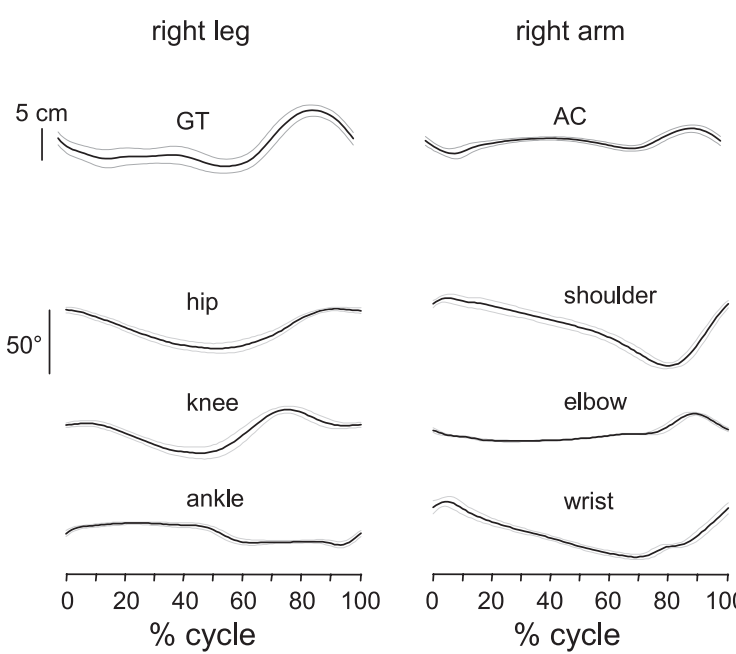
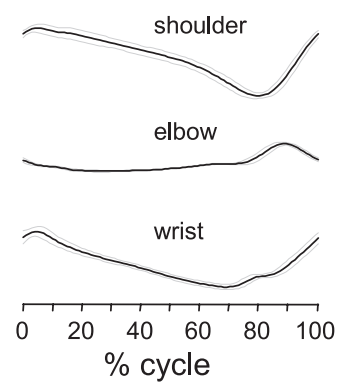

B
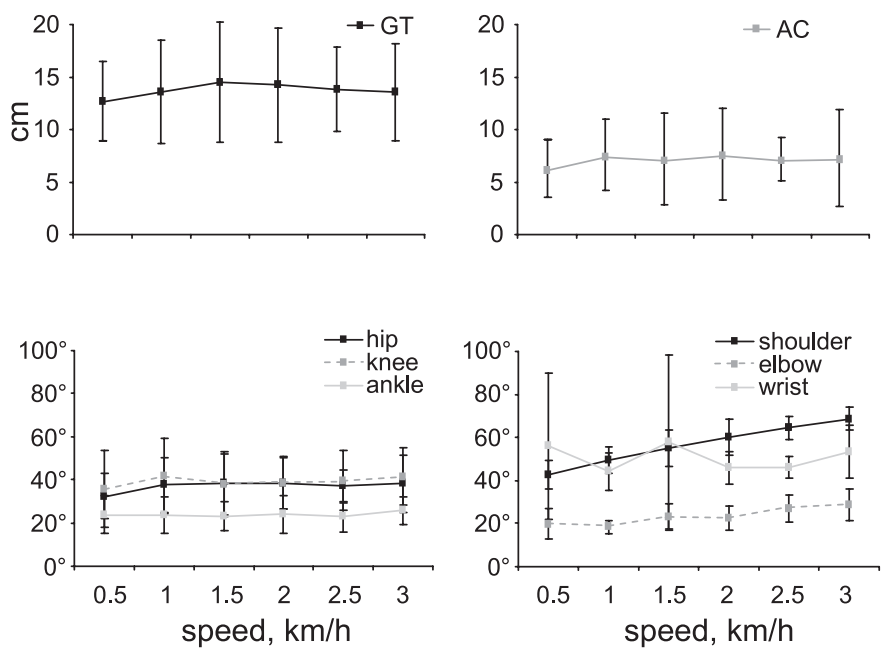

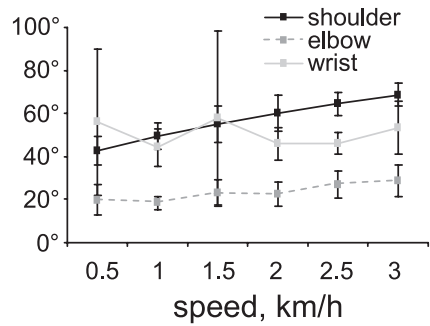

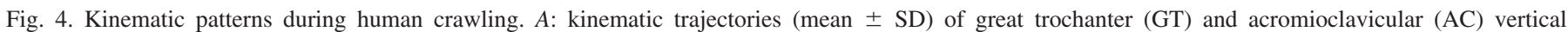
displacements and joint angles from a representative participant for a normalized gait cycle. $B$ : averages across participants ( \pm SD) for ranges of motion.

limb, but only the negative peak of the horizontal forces in the upper limb.

\section{EMG Activity and MN Activity}

Figure $6 A$ illustrates ensemble-averaged EMG waveforms through the stride cycle for crawling at increasing speeds. The largest differences in EMG activity relative to erect walking were in the upper limbs where there is a large burst of activity in the deltoids and triceps during upper limb stance. At the wrist, we saw that extensor activity increased during upper limb swing, whereas flexors exhibited a single burst at the end of upper limb stance. In the lower limbs, there was a trend for quadriceps (rectus femoris, vastus lateralis, vastus medialis) and tibialis anterior EMG activity to increase with crawling speed, whereas the burst in the ankle plantar flexors (lateral gastrocnemius, medial gastrocnemius, soleus) was diminished compared with upright walking.

With reference to cervical MN activity patterns in Fig. 6A, the activity was focused around the $\mathrm{C} 8$ and $\mathrm{T} 1$ segments during upper limb support with minimal activation during swing. The wrist muscle flexor burst at the end of upper limb stance is shown by activity in the C6-T1 segments. In the lumbosacral region (Fig. 6A, bottom), activity was focused around the L2-L4 segments immediately before and during lower limb stance. During swing, there was also a burst of activity around L4 and L5 that tended to increase with crawling speed. The

Table 1. Average limb orientation in mammals during quadrupedal walking

\begin{tabular}{lcc}
\hline \hline \multicolumn{1}{c}{ Group } & Average FL Angle, deg & Average HL Angle, deg \\
\hline Small mammals & $-6 \pm 5(6)$ & $3 \pm 7(6)$ \\
Primates & $-2 \pm 3(50)$ & $2 \pm 4(47)$ \\
Humans & $9 \pm 2 *(10)$ & $-21 \pm 2 \dagger(10)$ \\
\hline
\end{tabular}

Values are means $\pm \mathrm{SD}$; numbers in parentheses are the number of subjects. FL, forelimb, HL, hindlimb. Positive values denote limb protraction, zero refers to the vertical average limb orientation. The data for small mammals and primates are Larson and Stern (2009). The data on human crawling are presented at $3 \mathrm{~km} / \mathrm{h}$. A 1-tailed $t$-test was applied to assess significant differences from zero. ${ }^{*} P<0.0001 ; \dagger P<0.000001$. average activations over the stride cycle for the cervical and lumbosacral enlargements at each crawling speed are shown in Fig. 6B. Statistical analysis showed that MN activity increased significantly with crawling speed $\left[F_{(5,45)}=31.515, P<0.001\right]$ but did not differ significantly between cervical and lumbosacral enlargements $\left[F_{(1,9)}=0.268, P=0.62\right]$.

In general, the total $\mathrm{MN}$ activation level (and muscle activity) was considerably higher in crawling than in erect walking for both the cervical and lumbosacral enlargements (Fig. 6B). It is also worth noting that the participants verbally stated that the crawling protocol was much more fatiguing than normal walking.

Although temporal MN activation differed somewhat between participants (typical participants who crawled with lateral, no limb pairing, or diagonal patterns are shown in Fig. 7A), this was related to crawling behavior. With comparison of dominant MN patterns with crawling kinematics, a strong relationship $\left(r^{2}=0.866, P<0.001\right)$ was shown between the cervicallumbar spinal segment activity phase difference and IPL (Fig. $7 B$ ). It should be noted that two data points from a single participant were removed due to low levels of activation in the lumbar spinal segments.

\section{Effect of Changes in the Surface Inclination}

With increasing inclination of the crawling surface, we observed further changes in the EMG activity patterns (Fig. 8A). During upper limb stance, there was a decrease in triceps and posterior deltoid activity and even greater decreases in anterior deltoid activity as surface inclination increased. Although we observed these changes in shoulder musculature, the activation of wrist extensors during upper limb swing and the burst of wrist flexor activity at the end of upper limb stance remained in all conditions. In the lower limb, the quadriceps and tibialis anterior activity was similar at all inclinations, but there was a gradual appearance of gastrocnemius and soleus activity as inclination increased.

The changes in upper limb muscle activity are shown in terms of different MN burst patterns as surface inclination increased (Fig. 8A). In particular, cervical activity throughout 
A
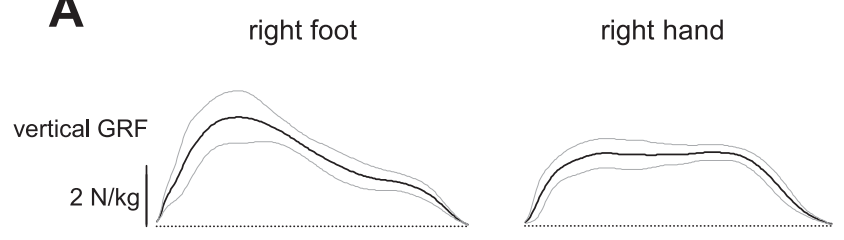

$1 \mathrm{~N} / \mathrm{kg}$

horizontal GRF

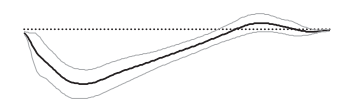

B
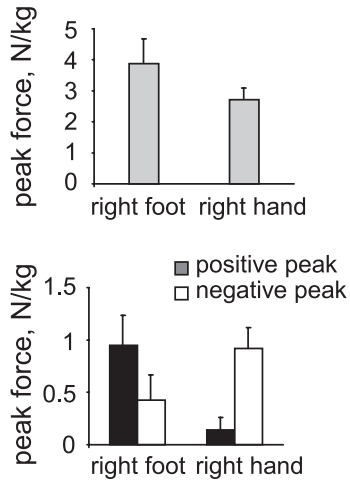
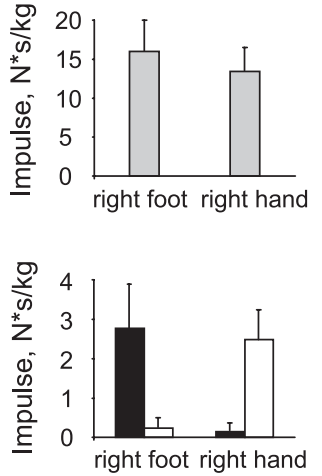

$\mathbf{C}$
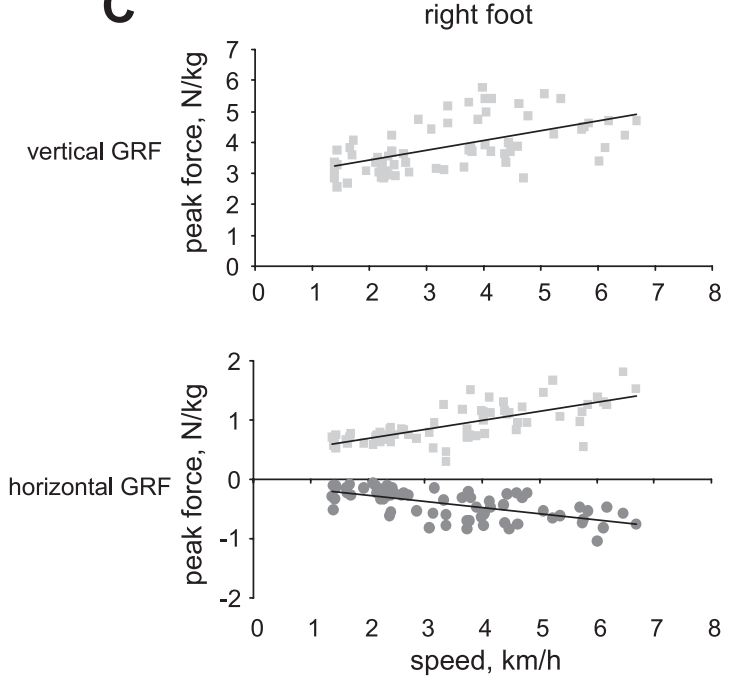

Fig. 5. Ground reaction force patterns during overground human crawling. A: ensemble-averaged ( $\pm \mathrm{SD}, n=9$ participants) vertical (top) and horizontal (bottom) ground reaction forces (GRF) for the lower (left) and upper limbs (right) during walking at a speed of $\sim 3.5 \mathrm{~km} / \mathrm{h}$. Positive GRF values correspond to the upward (vertical) and forward (horizontal) directions. B: peak GRF and impulses corresponding to the trials presented in $A$ (for horizontal forces and impulses, the absolute values are plotted). C: relationships between vertical (top) and horizontal (bottom) GRF and crawling velocity for the lower (left) and upper limbs (right). All GRF values were normalized to participant body weight. Each data point represents a single stride, and the line represents the best linear fit.

upper limb stance in the C8 and T1 segments diminished, with a burst of activation in the C6-T1 segments at the end of upper limb stance persisting with increasing surface inclination. In the lumbosacral region, activity in the L3 and L4 segments remained and there was an appearance of a burst in the $\mathrm{S} 1$ and S2 segments at the end of lower limb stance that increased with surface inclination (Fig. 8A, bottom). The average motoneuron activity throughout the stride is illustrated in Fig. $8 B$, and statistical analysis showed a surface inclination by spinal segment interaction effect $\left[F_{(6,30)}=15.784, P<0.001\right]$, suggesting an increase of sacral activity and a decrease in cervical activity as surface inclination increases.

\section{DISCUSSION}

This study specifically examined the spatiotemporal pattern of muscle activity, general spinal motor output characteristics, and kinematic and kinetic determinants of human crawling on hands and feet. Our results showed a number of similarities with quadrupedal locomotion in animals, but they also revealed several features specific to humans. Below we discuss our findings in the context of neuromuscular control of the lower and upper limbs and its comparison with animal locomotion.

\section{Motor Patterns in Human Crawling}

Specialized neural circuits in the caudal and rostral spinal cord organize the hindlimb and forelimb locomotor activity during animal locomotion (Grillner 2006; Kiehn 2006; Zehr et al. 2009). The coordination between cervical and lumbosacral pattern generation circuitry may be provided via mechanical coupling of arm-trunk-leg movements (Falgairolle et al. 2006; Pearson 2001) and via the long projecting propriospinal neurons coupling the cervical and lumbar spinal cord (Nathan et al. 1996). In this study we used an approach that provides information about CPG output in terms of segmental control rather than in terms of individual muscle control (Grasso et al. 2004; Ivanenko et al. 2006; Monaco et al. 2010; Yakovenko et al. 2002). Our results suggest that the temporal linkage between cervical and lumbosacral generators is flexible and gait dependent (Figs. 6-8). In this context, it is also noteworthy that cervical $\mathrm{MN}$ activity exhibits different timings during human 


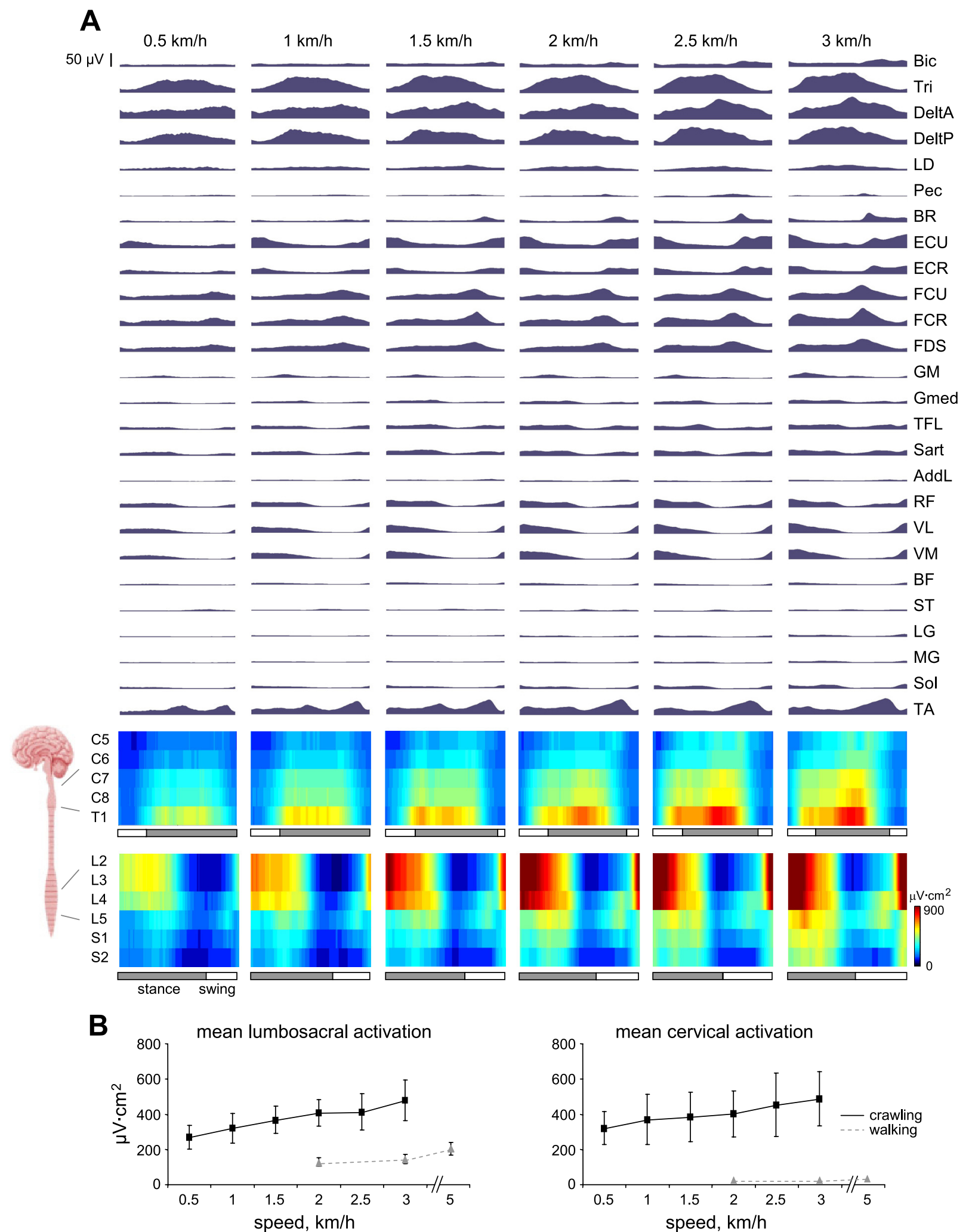

Fig. 6. Motor patterns in human crawling at different speeds. $A$ : ensemble-averaged patterns of electromyographic $($ EMG $)$ activity across participants $(n=10)$. Mean waveforms are plotted against the normalized gait cycle (beginning from the foot contact of the right leg). Corresponding $\alpha$-motoneuron ( $\alpha$-MN) activation patterns (normalized to physiological cross-sectional area method using Kendall's chart) of cervical and lumbosacral spinal enlargements are shown at the bottom of each panel. Bic, long head of biceps brachii; Tri, long head of triceps brachii; DeltA, anterior deltoid; DeltP, posterior deltoid; LD, latissimus dorsi; Pec, pectoralis major; BR, brachioradialis; ECU, extensor carpi ulnaris; ECR, extensor carpi radialis; FCU, flexor carpi ulnaris; FCR, flexor carpi radialis; FDS, flexor digitorium superficialis; GM, gluteus maximus; Gmed, gluteus medius; TFL, tensor fascia latte; Sart, sartorius; AddL, adductor longus; RF, rectus femoris; VL, vastus lateralis; VM, vastus medialis; ST, semitendinosis; BF, biceps femoris; LG, lateral gastrocnemius; MG, medial gastrocnemius; Sol, soleus; TA, tibialis anterior. $B$ : group means $( \pm \mathrm{SD})$ of lumbosacral (left) and cervical (right) activations from crawling and walking. 


\section{A}

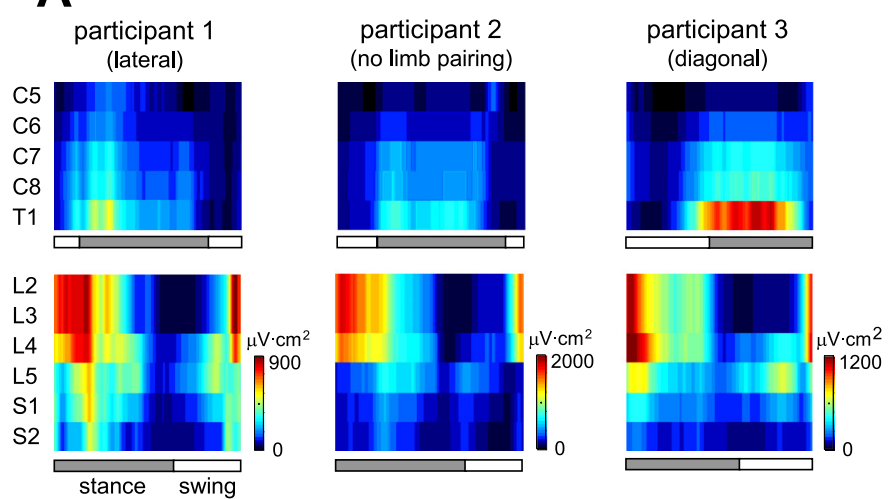

B

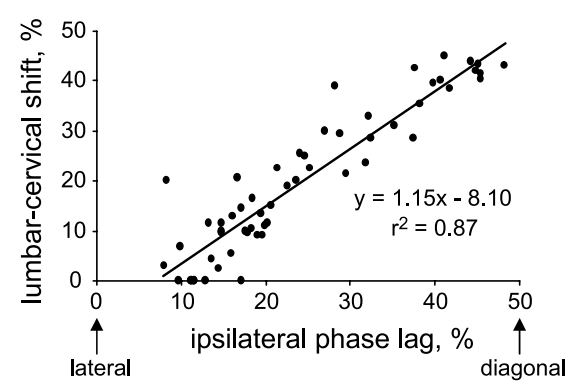

Fig. 7. Interparticipant variability of $\mathrm{MN}$ activity and crawling kinematics. A: MN activity of 3 separate participants walking at $1.5 \mathrm{~km} / \mathrm{h}$. These participants represent limb coordinations close to lateral, diagonal, and no limb pairing. $B$ : relationship between dominant lumbar-cervical $\mathrm{MN}$ activity phase shift (L2 + L3 vs. C8 + T1) and ipsilateral phase lag. Each data point represents the mean value for a participant for a single speed condition, and the line represents the estimated linear relationship between the variables.

walking vs. running (Ivanenko et al. 2008), likely due to differences in arm configuration and movements in these two gaits (flexed elbows during running). The coupling of the cervical and lumbosacral enlargements and the relative stance/ swing durations are gait and context dependent (Wannier et al. 2001), consistent with the neuromechanical tuning hypothesis according to which the phase durations generated by the CPGs closely match the required kinematics (Prochazka and Yakovenko 2007; Sylos Labini et al. 2011). Compared with habitual quadrupeds, human crawling may demand more cortical control due to the unfamiliar body posture requirements and/or intersegmental coordination. Furthermore, crawling experience may also contribute to the cortical control (Bell and Fox 1996).

The data in the literature on human crawling are rather limited. Webb et al. (2007) using harmonic analysis suggested that the arms behave like a pendulum in bipedal, but not quadrupedal, human locomotion. Swing phase in the upper limbs is shorter than in the lower limbs (Fig. 3A; results similar to those of Patrick et al. 2009), but this relationship does not hold when the inclination of the crawling surface is changed (Fig. 3B). Patrick et al. (2009) showed that from 0.4 to 1.1 cycles/s, a variety of IPLs (between diagonal and lateral) was utilized. Using the group mean, we confirmed this result, but at the same time we found that the individual data exhibit a large range of IPLs (the crawling of some participants was almost completely diagonal, whereas that of others was very close to lateral, Figs. 2 and 7). Similar ranges of IPL during human crawling have been noted throughout the literature (Gallagher et al. 2011; Patrick et al. 2009; Sparrow 1989; Sparrow and Newell 1994). Accordingly, the major locus of activity in the cervical segments (Fig. 6A) was slightly shifted to the left or right depending on whether the subject displayed more lateral or diagonal gait, respectively (Fig. 7). This variability is in sharp contrast with the stereotypy of erect walking kinematics and likely reflects the nonhabitual and nonecological character of crawling in human adults.

\section{Comparison With Cat and Monkey Locomotion}

Our findings revealed both similarities and differences between the human patterns and those of cat and monkey locomotion. Among the similarities, one may point out the alternating activity of cervical and lumbosacral enlargements, which reflects a diagonal nature of the interlimb coordination in primate locomotion (such as that observed in rhesus monkeys by Courtine et al. 2005), although humans exhibit great variability in interlimb phasing (Fig. 2). The relative swing duration and excursion also increased with speed (Fig. 3) as they do in rhesus monkeys (Courtine et al. 2005). Moreover, activation patterns in muscles such as vastus lateralis, rectus femoris, gluteus medius, sartorius, and tensor fascia latte displayed prolonged bursts during stance (Fig. 6A) relative to bipedal walking, but this prolonged pattern is similar to that in cats (Yakovenko et al. 2002) and monkeys (Courtine et al. 2005).

Although human crawling shares a number of common gait features with these animals, we found several critical differences in ground reaction forces and limb orientation and a reorganization of spinal segment motoneuron output. The double-humped pattern of vertical ground reaction force seen in cats (Lavoie et al. 1995) and in human erect walking (Winter 1991) is not observed in human crawling. The horizontal ground reaction force reveals predominantly negative and positive patterns in the upper and lower limbs, respectively (Fig. 5 ), consistent with the notion that the primary function of the upper limbs is to absorb energy to decelerate the body, whereas the lower limbs generate energy to progress the body forwards (Hanna et al. 2006). Nonhuman primates also show a more important propulsive role of the hindlimbs compared with the forelimbs (Kimura 1985), although to a much lesser extent than humans do (Fig. 5). Moreover, in human crawling, the lower limbs are retracted more than the hindlimbs in small mammals and primates (Table 1), presumably reflecting a relatively longer lower limb length and/or specific limb segment proportions in humans (Ivanenko et al. 2011; Leurs et al. 2011; Polk 2004).

Finally, a remarkable feature of human crawling is a lack of ankle plantar flexor muscle activation and, as a consequence, a relatively lower activation of sacral spinal segment output (Fig. 9, right). The spinal maps were constructed based on a large but incomplete sample of muscles because we were unable to record all muscles participating in the control of locomotion (e.g., some intrinsic foot muscles, although their PCSA is much smaller). A biomechanical reason for this may be due to the fact that the orientation of the foot is more vertical during crawling, likely because the anatomy of the human body (e.g., skeleton, relatively longer lower limb length and specific limb 
A

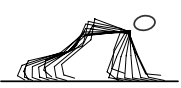

$0^{\circ}$
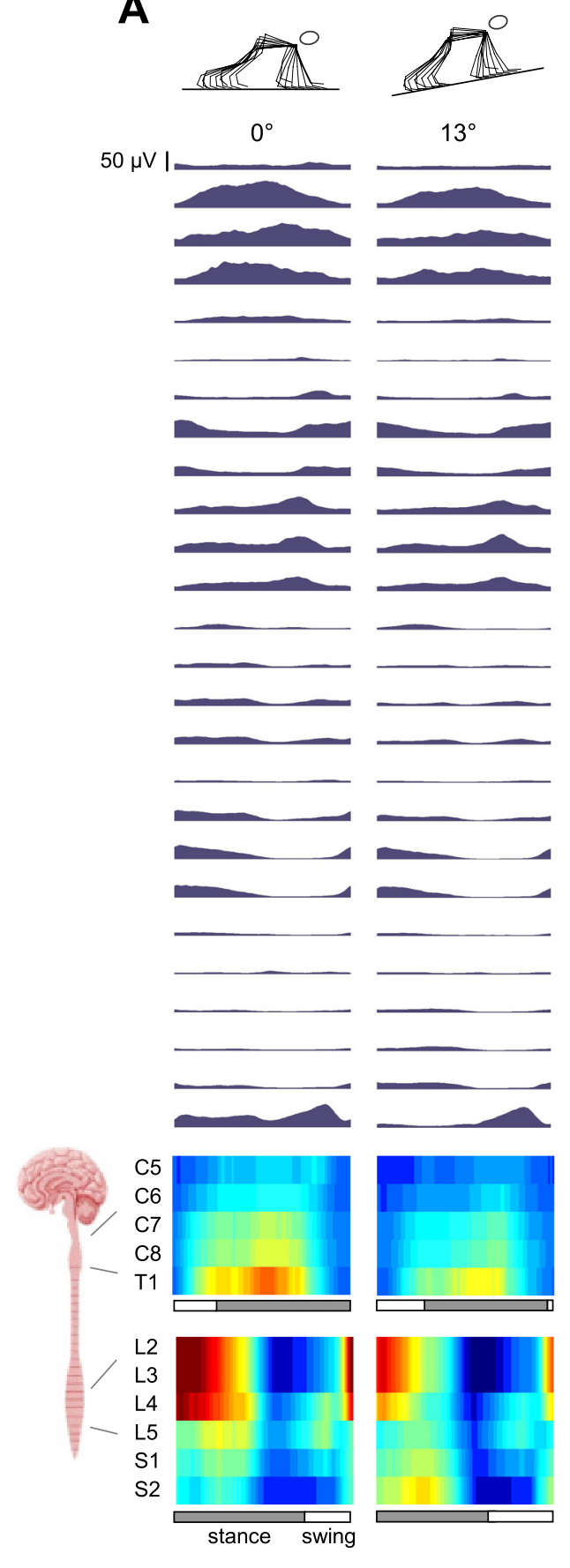

$13^{\circ}$

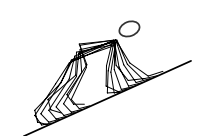

$27^{\circ}$
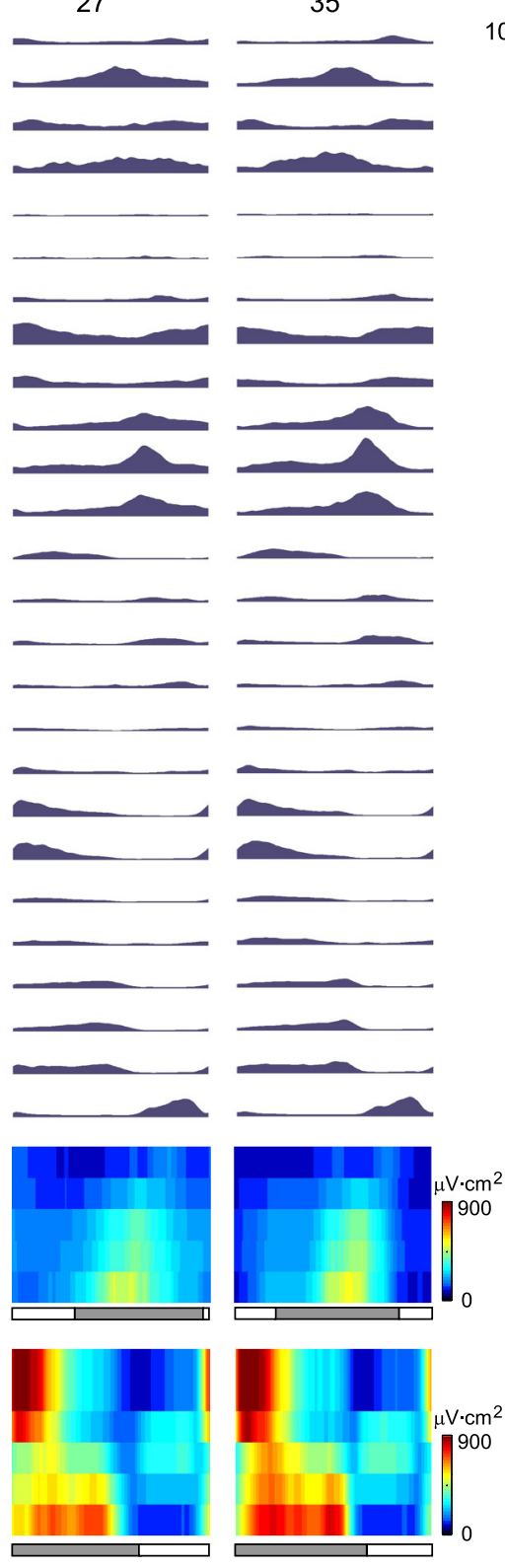

$35^{\circ}$

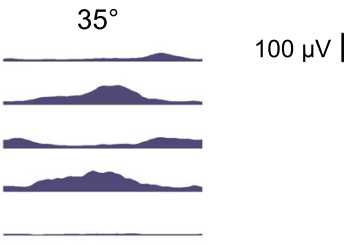

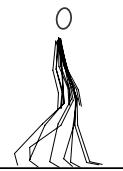

normal walking

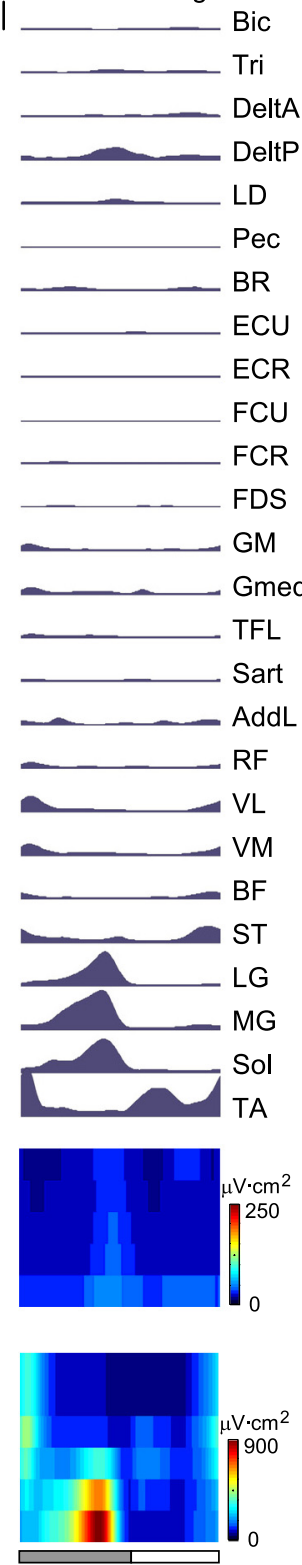

B
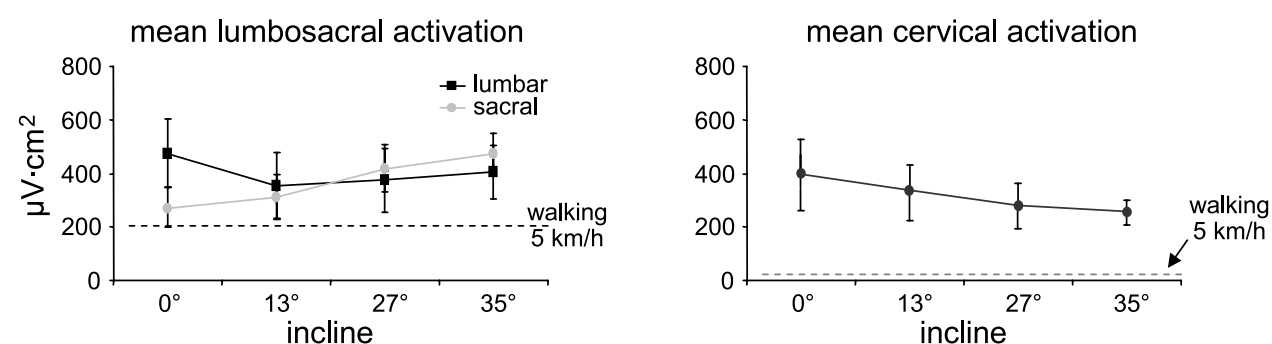

Fig. 8. Motor patterns in human crawling at different surface inclinations and during level upright walking at $5 \mathrm{~km} / \mathrm{h}$. The format of the presentation in $A$ is similar to that of Fig. 6 with a separate representation of lumbar and sacral activity in $B$. The dashed lines in $B$ represent mean lumbosacral and cervical $\alpha$-MN activation during upright walking at $5 \mathrm{~km} / \mathrm{h}$. 
A

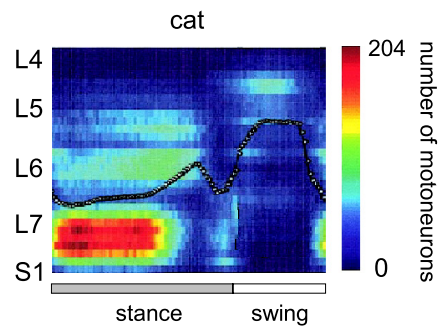

$150 \mu \mathrm{V}$

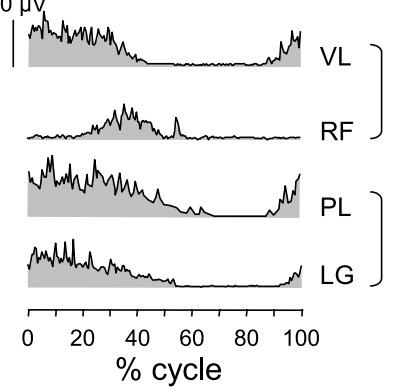

B

rhesus

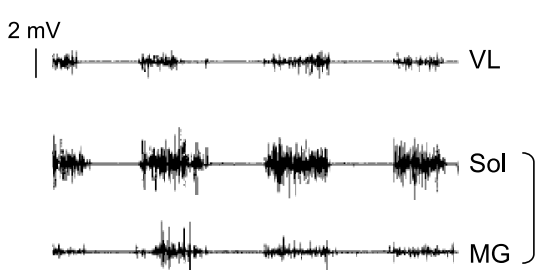

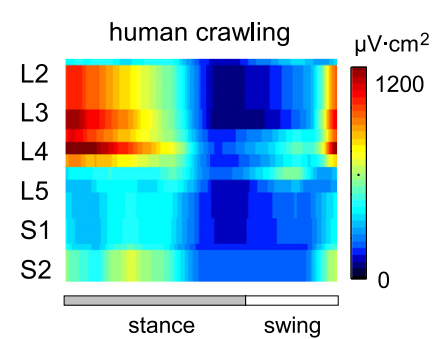

$50 \mu \mathrm{V}$
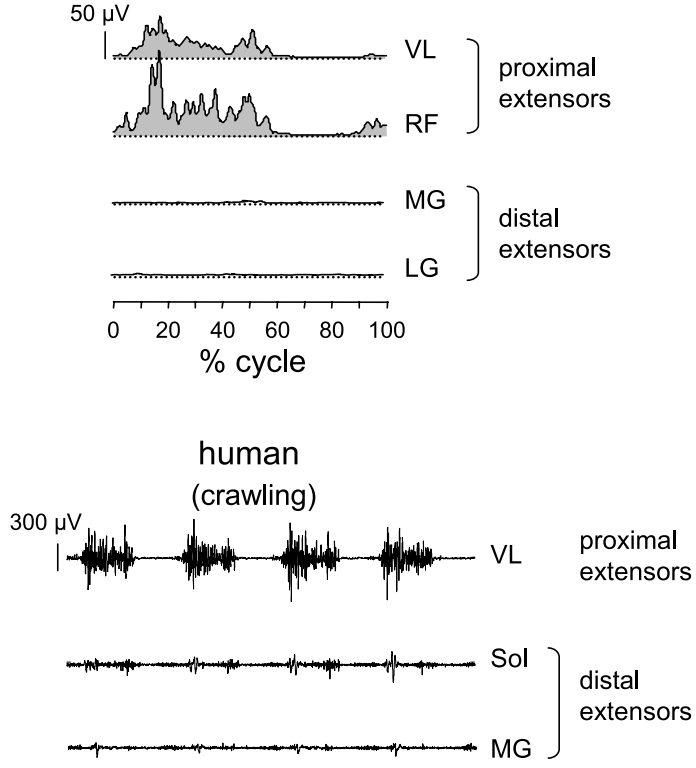

Fig. 9. Comparison of motor patterns in human crawling and quadrupedal animal (cat, monkey) locomotion. A, top left: spatiotemporal map in cat (modified from Yakovenko et al. 2002). A, top right: spatiotemporal map in human (normalized method, Sharrard's chart) is illustrated for 1 subject and corresponds to crawling at $3 \mathrm{~km} / \mathrm{h}$. A, bottom: EMG activity of VL, RF, MG, and LG muscles in the cat hindlimb (left; adapted from Carlson-Kuhta et al. 1998) and ipsilateral human leg $(r i g h t)$. PL, plantaris. For the purpose of illustration, human rectified EMGs were averaged across steps (25-Hz low-pass filtering). $B$ : examples of EMG activity in rhesus monkey (left; adapted from Recktenwald et al. 1999) and human crawling (right). segment proportions, muscle attachments, passive elasticity) is adapted to bipedal walking. Given the retracted limb orientation (Table 1), the ground reaction force vector may be closer to the ankle joint center, resulting in a decreased moment of force in the ankle joint. Nevertheless, a prominent activation of thigh muscles and a dominant activity of lumbar segments were present in human crawling at all speeds (Fig. 6). On the contrary, the available data in cats (Yakovenko et al. 2002) and monkeys (Courtine et al. 2005) indicate a more uniform redistribution of proximal and distal extensor activities and a prominent activation of the caudal spinal segments (Fig. 9, left). It should be stressed that the quadrupedal mode of walking we investigated in this study is, of course, different from that exhibited by gorillas and chimpanzees, which walk on their knuckles, and from orangutans, which walk on their fists.

In addition, several research groups have trained monkeys to walk with a bipedal gait pattern (Fitzsimmons et al. 2009; Hirasaki et al. 2004; Nakajima et al. 2004). From the energetic point of view, nonhuman primates trained to walk bipedally consume less metabolic energy during quadrupedal than bipedal gait (Nakatsukasa et al. 2004), whereas humans display considerably higher levels of mean MN activation in both cervical and lumbosacral enlargements during crawling compared with walking (Fig. 6B), supporting the idea of a high specialization of the neuromuscular apparatus and body proportions to specific gaits (Alexander 2004; Ivanenko et al. 2011; Leurs et al. 2011; Polk 2004; Sockol et al. 2007).

\section{Crawling at Different Inclines}

We assessed motor patterns for three levels of upslope crawling and compared them with similar data for level treadmill crawling and walking (Fig. 8). The principal purpose of this upslope crawling paradigm was to gain insight about the functions of muscles and the mechanisms of neural control required for different body orientations and support surfaces. Also, crawling at increasing inclines represents a gradual transition toward the upright body orientation and thus an "intermediate" form of locomotion between quadrupedal and erect bipedal walking.

Although these similarities exist, fundamental differences are also evident. As surface inclination increases, there is a decrease in cervical $\alpha$-MN activity that leads to an overall decrement of upper limb muscle activity (Fig. 8B, right). More importantly, we see the appearance of a sacral $\alpha$-MN burst and increased ankle plantar flexor muscle activation (Fig. 8, left), likely related to the change in direction of the ground reaction force relative to the ankle joint (due to the more horizontal foot segment orientation compared with level crawling, see above), leading to a larger moment of force. The reason for these changes may be directly related to the redistribution of body weight between the upper and lower limbs and across lower limb joints.

\section{CONCLUSIONS}

Overall, this study provides new data on the reorganization and adaptation of the output of central pattern generators 
involved in the control of quadrupedal human locomotion as a function of different environmental constraints. Of particular interest was a decrease in sacral compared with lumbar motoneuron activation and the flexibility in the spatiotemporal output for the spinal segments. Sacral activity tended to increase with increasing surface inclination, more closely resembling upright walking. The findings of neuromechanical coupling between upper and lower limb motion could lead to a better understanding of the hypothetical evolution of human gait from quadrupedal locomotion (Schmitt 2003; Thorpe et al. 2007; Zampagni et al. 2011) and also may be of particular interest in the construction of biologically inspired robots (Ijspeert 2008; Pfeifer et al. 2007) or for clinical applications (Ferris et al. 2006; Klimstra et al. 2009; Tan 2010).

\section{GRANTS}

This work was supported by a CRUSOE grant from the Italian Space Agency and by MINDWALKER Grant 247959 and AMARSi Grant 248311 from the European Union FP7-ICT Program.

\section{DISCLOSURES}

No conflicts of interest, financial or otherwise, are declared by the author(s).

\section{AUTHOR CONTRIBUTIONS}

Author contributions: M.J.M., Y.P.I., and F.L. conception and design of research; M.J.M., Y.P.I., G.C., F.S.L., and F.L. performed experiments; M.J.M., Y.P.I., G.C., F.S.L., and F.L. analyzed data; M.J.M., Y.P.I., G.C., F.S.L., and F.L. interpreted results of experiments; M.J.M., Y.P.I., and F.L. prepared figures; M.J.M., Y.P.I., and F.L. drafted manuscript; M.J.M., Y.P.I., G.C., F.S.L., and F.L. edited and revised manuscript; M.J.M., Y.P.I., G.C., F.S.L., and F.L. approved final version of manuscript.

\section{REFERENCES}

Alexander RM. Bipedal animals, and their differences from humans. J Anat 204: 321-330, 2004

Babic $^{`}$ J, Karčnik T, Bajd T. Stability analysis of four-point walking. Gait Posture 14: 56-60, 2001.

Bell MA, Fox NA. Crawling experience is related to changes in cortical organization during infancy: evidence from EEG coherence. Dev Psychobiol 29: 551-561, 1996.

Cappellini G, Ivanenko YP, Dominici N, Poppele RE, Lacquaniti F. Migration of motor pool activity in the spinal cord reflects body mechanics in human locomotion. J Neurophysiol 104: 3064-3073, 2010.

Carlson-Kuhta P, Trank TV, Smith JL. Forms of forward quadrupedal locomotion. II. A comparison of posture, hindlimb kinematics, and motor patterns for upslope and level walking. J Neurophysiol 79: 1687-1701, 1998.

Courtine G, Roy RR, Hodgson J, McKay H, Raven J, Zhong H, Yang H, Tuszynski MH, Edgerton VR. Kinematic and EMG determinants in quadrupedal locomotion of a non-human primate (Rhesus). J Neurophysiol 93: 3127-3145, 2005.

Crenna P, Carpinella I, Lopiano L, Marzegan A, Rabuffetti M, Rizzone M, Lanotte M, Ferrarin M. Influence of basal ganglia on upper limb locomotor synergies. Evidence from deep brain stimulation and L-DOPA treatment in Parkinson's disease. Brain 131: 3410-3420, 2008.

de Sèze M, Falgairolle M, Viel S, Assaiante C, Cazalets JR. Sequential activation of axial muscles during different forms of rhythmic behavior in man. Exp Brain Res 185: 237-247, 2008.

Dietz V. Do human bipeds use quadrupedal coordination? Trends Neurosci 25 : 462-467, 2002.

Dietz V, Michel J. Human bipeds use quadrupedal coordination during locomotion. Ann NY Acad Sci 1164: 97-103, 2009.

Donker SF, Mulder T, Nienhuis B, Duysens J. Adaptations in arm movements for added mass to wrist or ankle during walking. Exp Brain Res 146: 26-31, 2002.
Falgairolle M, de Sèze M, Juvin L, Morin D, Cazalets JR. Coordinated network functioning in the spinal cord: an evolutionary perspective. $J$ Physiol (Paris) 100: 304-316, 2006.

Ferris DP, Huang HJ, Kao PC. Moving the arms to activate the legs. Exerc Sport Sci Rev 34: 113-120, 2006.

Fitzsimmons NA, Lebedev MA, Peikon ID, Nicolelis MA. Extracting kinematic parameters for monkey bipedal walking from cortical neuronal ensemble activity. Front Integr Neurosci 3: 3, 2009.

Gallagher S, Pollard J, Porter WL. Locomotion in restricted space: kinematic and electromyographic analysis of stoopwalking and crawling. Gait Posture 33: 71-76, 2011.

Getchell N, Forrester L, Whitall J. Individual differences and similarities in the stability, timing consistency, and natural frequency of rhythmic coordinated actions. Res $Q$ Exerc Sport 72: 13-21, 2001.

Grasso R, Ivanenko YP, Zago M, Molinari M, Scivoletto G, Castellano V, Macellari V, Lacquaniti F. Distributed plasticity of locomotor pattern generators in spinal cord injured patients. Brain 127: 1019-1034, 2004.

Grillner S. Biological pattern generation: the cellular and computational logic of networks in motion. Neuron 52: 751-766, 2006.

Hanna JB, Polk JD, Schmitt D. Forelimb and hindlimb forces in walking and galloping primates. Am J Phys Anthropol 130: 529-535, 2006.

Hildebrand M. Symmetrical gaits of primates. Am J Phys Anthropol 26: 119-130, 1967.

Hirasaki E, Ogihara N, Hamada Y, Kumakura H, Nakatsukasa M. Do highly trained monkeys walk like humans? A kinematic study of bipedal locomotion in bipedally trained Japanese macaques. J Hum Evol 46: 739$750,2004$.

Ijspeert AJ. Central pattern generators for locomotion control in animals and robots: a review. Neural Netw 21: 642-653, 2008.

Ivanenko YP, Cappellini G, Dominici N, Poppele RE, Lacquaniti F. Coordination of locomotion with voluntary movements in humans. $\mathrm{J} \mathrm{Neu}$ rosci 25: 7238-7253, 2005.

Ivanenko YP, Poppele RE, Lacquaniti F. Spinal cord maps of spatiotemporal $\alpha$-motoneuron activation in humans walking at different speeds. $J$ Neurophysiol 95: 602-618, 2006.

Ivanenko YP, Cappellini G, Poppele RE, Lacquaniti F. Spatiotemporal organization of alpha-motoneuron activity in the human spinal cord during different gaits and gait transitions. Eur J Neurosci 27: 3351-3368, 2008.

Ivanenko YP, Dominici N, Daprati E, Nico D, Cappellini G, Lacquaniti F. Locomotor body scheme. Hum Mov Sci 30: 341-351, 2011.

Kendall FP, McCreary EK, Provance PG. Muscles. Testing and Function. Baltimore, MD: Williams and Wilkins, 1993.

Kiehn O. Locomotor circuits in the mammalian spinal cord. Annu Rev Neurosci 29: 279-306, 2006.

Kimura T. Bipedal and quadrupedal locomotion of primates; comparative dynamics. In: Primate Morphophysiology: Locomotor Analyses and Human Bipedalism, edited by Kondo S. Tokyo: University of Tokyo Press, 1985, p. 81-104.

Klimstra MD, Thomas E, Stoloff RH, Ferris DP, Zehr EP. Neuromechanical considerations for incorporating rhythmic arm movement in the rehabilitation of walking. Chaos 19: 026102, 2009.

Larson SG, Stern JT Jr. Hip extensor EMG and forelimb/hind limb weight support asymmetry in primate quadrupeds. Am J Phys Anthropol 138: 343-355, 2009.

Lavoie S, McFadyen B, Drew T. A kinematic and kinetic analysis of locomotion during voluntary gait modification in the cat. Exp Brain Res 106: 39-56, 1995.

Leurs F, Ivanenko YP, Bengoetxea A, Cebolla AM, Dan B, Lacquaniti F, Cheron GA. Optimal walking speed following changes in limb geometry. $J$ Exp Biol 214: 2276-2282, 2011.

Monaco V, Ghionzoli A, Micera S. Age-related modifications of muscle synergies and spinal cord activity during locomotion. J Neurophysiol 104: 2092-2102, 2010.

Nakajima K, Mori F, Takasu C, Mori M, Matsuyama K, Mori S. Biomechanical constraints in hindlimb joints during the quadrupedal versus bipedal locomotion of M. fuscata. Prog Brain Res 143: 183-190, 2004.

Nakatsukasa M, Ogihara N, Hamada Y, Goto Y, Yamada M, Hirakawa T, Hirasaki E. Energetic costs of bipedal and quadrupedal walking in Japanese macaques. Am J Phys Anthropol 124: 248-256, 2004.

Nathan PW, Smith M, Deacon P. Vestibulospinal, reticulospinal and descending propriospinal nerve fibres in man. Brain 119: 1809-1833, 1996.

Patrick SK, Noah JA, Yang JF. Interlimb coordination in human crawling reveals similarities in development and neural control with quadrupeds. $J$ Neurophysiol 101: 603-613, 2009. 
Pearson KG. Could enhanced reflex function contribute to improving locomotion after spinal cord repair? J Physiol 533: 75-81, 2001.

Pfeifer R, Lungarella M, Iida F. Self-organization, embodiment, and biologically inspired robotics. Science 318: 1088-1093, 2007.

Polk JD. Influences of limb proportions and body size on locomotor kinematics in terrestrial primates and fossil hominins. J Hum Evol 47: 237-252, 2004.

Prochazka A, Yakovenko S. Predictive and reactive tuning of the locomotor CPG. Integr Comp Biol 47: 474-481, 2007.

Ramsay JW, Hunter BV, Gonzalez RV. Muscle moment arm and normalized moment contributions as reference data for musculoskeletal elbow and wrist joint models. J Biomech 42: 463-473, 2009.

Recktenwald MR, Hodgson JA, Roy RR, Riazanski S, McCall GE, Kozlovskaya I, Washburn DA, Fanton JW, Edgerton VR. Effects of spaceflight on rhesus quadrupedal locomotion after return to $1 \mathrm{G}$. J Neurophysiol 81: 2451-2463, 1999.

Schmitt D. Insights into the evolution of human bipedalism from experimental studies of humans and other primates. J Exp Biol 206: 1437-1448, 2003.

Sharrard WJW. The segmental innervation of the lower limb muscles in man. Ann R Coll Surg Engl 35: 106-122, 1964.

Sockol MD, Raichlen DA, Pontzer H. Chimpanzee locomotor energetics and the origin of human bipedalism. Proc Natl Acad Sci USA 104: 1226512269, 2007.

Sparrow WA. Creeping patterns of human adults and infants. Am J Phys Anthropol 78: 387-401, 1989.

Sparrow WA, Newell KM. The coordination and control of human creeping with increases in speed. Behav Brain Res 63: 151-158, 1994

Sylos Labini F, Ivanenko YP, Cappellini G, Gravano S, Lacquaniti F. Smooth changes in the EMG patterns during gait transitions under body weight unloading. J Neurophysiol 106: 1525-1536, 2011.
Tan U. Uner Tan syndrome: history, clinical evaluations, genetics, and the dynamics of human quadrupedalism. Open Neurol J 4: 78-89, 2010.

Thorpe SK, Holder RL, Crompton RH. Origin of human bipedalism as an adaptation for locomotion on flexible branches. Science 316: 1328-1331, 2007.

Wannier T, Bastiaanse C, Colombo G, Dietz V. Arm to leg coordination in humans during walking, creeping and swimming activities. Exp Brain Res 141: 375-359, 2001.

Ward SR, Eng CM, Smallwood LH, Lieber RL. Are current measurements of lower extremity muscle architecture accurate? Clin Orthop Relat Res 467: 1074-1082, 2009.

Webb D, Sparrow WA. Description of joint movements in human and non-human primate locomotion using Fourier analysis. Primates 48: $277-$ 292, 2007.

Winter DA. The Biomechanics and Motor Control of Human Gait: Normal, Elderly and Pathological. Waterloo, ON, Canada: Waterloo Biomechanics, 1991.

Wood JE, Meek SG, Jacobsen SC. Quantitation of human shoulder anatomy for prosthetic arm control. I. Surface modelling. J Biomech 22: 273-292, 1989.

Yakovenko S, Mushahwar V, VanderHorst V, Holstege G, Prochazka A. Spatiotemporal activation of lumbosacral motoneurons in the locomotor step cycle. J Neurophysiol 87: 1542-1553, 2002.

Yokochi K, Hosoe A, Shimabukuro S, Kodama K. Gross motor patterns in children with cerebral palsy and spastic diplegia. Pediatr Neurol 6: 245250, 1990.

Zampagni ML, Brigadoi S, Schena F, Tosi P, Ivanenko YP. Idiosyncratic control of the center of mass in expert climbers. Scand J Med Sci Sports 21: 688-699, 2011.

Zehr EP, Hundza SR, Vasudevan EV. The quadrupedal nature of human bipedal locomotion. Exerc Sport Sci Rev 37: 102-108, 2009.

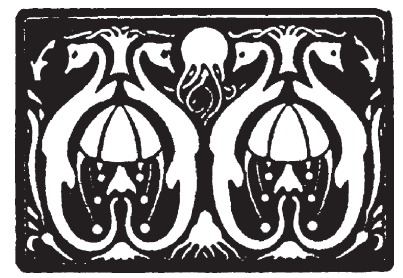

